IFT-25-03 FUT-03-02

UCRHEP-T364

TOKUSHIMA Report

(hep-ph/0310159)

\title{
Probing Anomalous Top-Quark Couplings Induced by Dim.6 Operators at Photon Colliders
}

\author{
Bohdan GRZADKOWSKI ${ }^{1), a)}$, ZenRō HIOKI ${ }^{2),}$, , \\ Kazumasa OHKUMA ${ }^{3), c)}$ and José WUDKA ${ }^{4), d)}$ \\ 1) Institute of Theoretical Physics, Warsaw University \\ Hoża 69, PL-00-681 Warsaw, POLAND \\ and \\ CERN, Department of Physics \\ Theory Division \\ 1211 Geneva 23, Switzerland \\ 2) Institute of Theoretical Physics, University of Tokushima \\ Tokushima 770-8502, JAPAN \\ 3) Department of Management Science, Fukui University of Technology \\ Fukui 910-8505, JAPAN \\ 4) Department of Physics, University of California \\ Riverside CA 92521-0413, USA
}

\begin{abstract}
Possible anomalous top-quark couplings induced by $S U(2) \times U(1)$ gauge-invariant dimension- 6 effective operators were studied in the process of $t \bar{t}$ productions and decays at polarized $\gamma \gamma$ colliders. Two $C P$-violating asymmetries, a linearpolarization asymmetry and a circular-polarization asymmetry, were computed including both non-standard $t \bar{t} \gamma$ and $\gamma \gamma H$ couplings. An optimal-observable analysis for the process $\gamma \gamma \rightarrow t \bar{t} \rightarrow \ell^{ \pm} \ldots$ was performed in order to estimate the precision for determination of all relevant non-standard couplings, including the anomalous tbW coupling.
\end{abstract}

PACS: 14.60.-z, 14.65.Ha, 14.70.Bh

Keywords: anomalous top-quark couplings, $\gamma \gamma$ colliders

\footnotetext{
a) E-mail address: bohdan.grzadkowski@fuw.edu.pl

b) E-mail address: hioki@ias.tokushima-u.ac.jp

c) E-mail address: ohkuma@ccmails.fukui-ut.ac.jp

d) E-mail address: jose.wudka@ucr . edu
} 


\section{Introduction}

A lot of data have been accumulated on the top-quark ever since its discovery [1]. However, it still remains an open question whether the top-quark couplings obey the Standard-Model (SM) scheme of the electroweak forces or there exists a contribution from some non-standard physics. Next-generation $e^{+} e^{-}$linear colliders are expected to work as top-quark factories, and therefore a lot of attention has been paid to study top-quark interactions through $e \bar{e} \rightarrow t \bar{t}$ (see, e.g., [2, 3] and their reference lists).

An interesting option for $e^{+} e^{-}$machines could be that of photon-photon collisions, where initial energetic photons are produced through electron and laser-light backward scattering 4, 5]; such a collider presents remarkable advantages for the study of $C P$ violation. In the case of $e \bar{e}$ collisions, the only initial states that are

relevant are $C P$-even states $\left|e_{L / R} \bar{e}_{R / L}\right\rangle$ under the usual assumption that the electron mass can be neglected and that the leading contributions to $t \bar{t}$ production come from $s$-channel vector-boson exchanges. Therefore all $C P$-violating observables must be constructed from final-particle momenta/polarizations. In contrast, a $\gamma \gamma$ collider offers a unique possibility of preparing the polarization of the incident photon beams, which can be used to construct $C P$-violating asymmetries without relying on final-state information.

This is why a number of authors have considered top-quark production and decays in $\gamma \gamma$ collisions in order to study i) Higgs-boson couplings to the top-quark and photon [6]-[12], or ii) anomalous top-quark couplings to photon [13]-[15]. However, what is supposed to be observed in real experiments are combined signals that originate both from the process of top-quark production, and in addition, from its decays. In this sense, we have to conclude that in general those previous papers are not realistic enough. Therefore here we will consider $\gamma \gamma \rightarrow t \bar{t} \rightarrow \ell^{ \pm} X$ including all possible non-standard interactions together (production and decay), and perform comprehensive analysis as model-independently as possible within the effective-Lagrangian framework of Buchmüller and Wyler [16].

The paper is organized as follows. In sec.2 we will briefly describe a framework for our effective-Lagrangian approach. Section 3 will be devoted to determination of 
the operator set relevant for the $t \bar{t}$ production/decay processes, and the Feynman rules they induce. Based on them we compute two polarization asymmetries in sec.4, and carry out an optimal-observable analysis aiming to determine all the unknown parameters simultaneously in sec.5. Then we summarize our results in the final section. For completeness, in the appendix we collect formulas that are needed to describe the Stokes parameters for the initial photon beams. There we also present some other formulas which are necessary for the calculation of the cross sections $\sigma\left(\gamma \gamma \rightarrow t \bar{t} \rightarrow \ell^{ \pm} X\right)$. Throughout this work, we use FORM [17] for main algebraic calculations.

\section{Framework}

In this article we use a model-independent technique based on effective low-energy Lagrangian [16, 18] to describe possible new-physics effects. In this approach, we are supposed to consider the SM Lagrangian modified by the addition of a series of $S U(2) \times U(1)$ gauge-invariant operators whose coefficients parameterize the lowenergy effects of the underlying high-scale physics.

Assuming that the heavy degrees of freedom decouple implies that the effective operators have coefficients suppressed by appropriate inverse powers of $\Lambda$, where $\Lambda$ expresses the energy scale of new physics [19]. If $\Lambda \gg v \sim 250 \mathrm{GeV}$ then the leading effects are generated by operators of mass-dimension 6 (the dimension 5 operator violates the lepton-number conservation and therefore it is irrelevant hereafter) [16]:

$$
\mathcal{L}_{\text {eff }}=\mathcal{L}_{\mathrm{SM}}+\frac{1}{\Lambda^{2}} \sum_{i}\left(\alpha_{i} \mathcal{O}_{i}+\text { h.c. }\right) .
$$

If the high-scale theory is a weakly-coupled gauge theory, one can show that coefficients $\alpha_{i}$ of the operators that may be generated at the tree level of the underlying theory could be $\mathcal{O}(1)$ while those that can appear only at the one-loop level of perturbative expansion must be suppressed by at least the loop-factor $1 /(4 \pi)^{2}[18$. Therefore we will assume all the couplings except those from the SM tree level are small and take into account neither contributions of order $1 / \Lambda^{n}$ with $n>2$ nor the SM higher-order terms. Below we will refer to application of $\mathcal{L}_{\text {eff }}$ as to "B\&W" scenario [16]. Given our emphasis on top-quark physics the effects of the first two 
fermion generations will be ignored.

In our framework, certain types of anomalous interactions are not included. For instance, $\gamma \gamma Z$ couplings, which has been studied in [20], is one of those couplings since it is not on the list of $S U(3) \times S U(2) \times U(1)$ invariant dim.6 operators (such an operator will appear with a suppression factor of $1 / \Lambda^{4}$ and can be ignored). An extended $t \bar{t} H$ coupling is not taken into account either, since the other end of the Higgs propagator in $\gamma \gamma \rightarrow H \rightarrow t \bar{t}$ is a pure non-standard $\gamma \gamma H$ vertex, which requires that the $t \bar{t} H$ coupling should come from the SM tree level within our approximation.

Before going to the next section, there is an important comment in order. Even though our approach is model independent, one should keep in mind that we assume $\Lambda \gg v$. Consequently for the process considered here our results should not be directly applicable in the context of, e.g., two Higgs-doublet model with extra scalar bosons having their masses of $\mathcal{O}(v)$. That kind of non-standard interactions would lead to non-local form-factors that cannot be accommodated within our present framework. This is because of the virtual and therefore off-shell top-quark line that is present in the amplitudes for $\gamma \gamma \rightarrow t \bar{t}$. Note that this is quite in contrast to $e \bar{e} \rightarrow \gamma / Z \rightarrow t \bar{t}$ case, where for a given $\sqrt{s}$ we are able to write down the most general invariant amplitude with constant form factors because all the kinematic variables are fixed.

\section{Anomalous couplings from dim.6 operators}

Within the B\&W scenario, the following dim.6 operators could contribute to the continuum top-quark production process $\gamma \gamma \rightarrow t \bar{t}$ :

$$
\begin{array}{ll}
\mathcal{O}_{u B}=i \bar{u} \gamma_{\mu} D_{\nu} u B^{\mu \nu}, & \mathcal{O}_{q B}=i \bar{q} \gamma_{\mu} D_{\nu} q B^{\mu \nu}, \\
\mathcal{O}_{q W}=i \bar{q} \tau^{i} \gamma_{\mu} D_{\nu} q W^{i \mu \nu}, & \mathcal{O}_{u B}^{\prime}=\left(\bar{q} \sigma^{\mu \nu} u\right) \tilde{\varphi} B_{\mu \nu}, \\
\mathcal{O}_{u W}=\left(\bar{q} \sigma^{\mu \nu} \tau^{i} u\right) \tilde{\varphi} W_{i \mu \nu}, &
\end{array}
$$

where we have adopted the notation from ref.[16]. Each of the above operators contains both $C P$-violating and $C P$-conserving parts.

These operators, however, are not independent. Using the Bianchi identities and SM equations of motion we find:

$$
\mathcal{O}_{u B}=-\frac{1}{4}\left[i \Gamma_{u}\left(\mathcal{O}_{u B}^{\prime}+g^{\prime} \mathcal{O}_{u \varphi}\right)+\text { h.c. }\right]-i \frac{g^{\prime}}{2} \mathcal{O}_{\varphi u}
$$




$$
\begin{aligned}
& +4 \text {-fermion operators }+ \text { total derivative } \\
\mathcal{O}_{q B} & =\frac{1}{4}\left[i \Gamma_{u}\left(\mathcal{O}_{u B}^{\prime}+g^{\prime} \mathcal{O}_{u \varphi}\right)+i \Gamma_{d}\left(\mathcal{O}_{d B}^{\prime}+g^{\prime} \mathcal{O}_{d \varphi}\right)+\text { h.c. }\right] \\
& -i \frac{g^{\prime}}{2} \mathcal{O}_{\varphi q}^{(1)}+4 \text {-fermion operators }+ \text { total derivative } \\
\mathcal{O}_{q W} & =\frac{1}{4}\left[i \Gamma_{u}\left(\mathcal{O}_{u W}-g \mathcal{O}_{u \varphi}\right)+i \Gamma_{d}\left(\mathcal{O}_{d W}+g \mathcal{O}_{d \varphi}\right)+\text { h.c. }\right] \\
& -i \frac{g}{2} \mathcal{O}_{\varphi q}^{(3)}+4 \text {-fermion operators }+ \text { total derivative }
\end{aligned}
$$

where $\Gamma_{u, d}$ are the Yukawa couplings for up- and down-type quarks, respectively. ${ }^{\sharp 1}$ These relations imply that the operators $\mathcal{O}_{u B}, \mathcal{O}_{q B}$ and $\mathcal{O}_{q W}$ are redundant and can be dropped, which means the set of relevant operators is reduced to

$$
\mathcal{O}_{u B}^{\prime}=\left(\bar{q} \sigma^{\mu \nu} u\right) \tilde{\varphi} B_{\mu \nu}, \quad \mathcal{O}_{u W}=\left(\bar{q} \sigma^{\mu \nu} \tau^{i} u\right) \tilde{\varphi} W_{i \mu \nu} .
$$

This reduction is important, as it allows to determine the minimal set of operators that are relevant for the process considered here. In practice, it can drastically decrease the effort that otherwise would be necessary to obtain the final and correct result. In particular, it shows that the contact interactions of the type $t \bar{t} \gamma \gamma$ should be dropped.

On the other hand, the following operators contribute to $\gamma \gamma \rightarrow t \bar{t}$ through the resonant $s$-channel Higgs-boson exchange:

$$
\begin{array}{ll}
\mathcal{O}_{\varphi \tilde{W}}=\left(\varphi^{\dagger} \varphi\right) \tilde{W}_{\mu \nu}^{i} W^{i \mu \nu}, & \mathcal{O}_{\varphi W}=\left(\varphi^{\dagger} \varphi\right) W_{\mu \nu}^{i} W^{i \mu \nu} / 2 \\
\mathcal{O}_{\varphi \tilde{B}}=\left(\varphi^{\dagger} \varphi\right) \tilde{B}_{\mu \nu} B^{\mu \nu}, & \mathcal{O}_{\varphi B}=\left(\varphi^{\dagger} \varphi\right) B_{\mu \nu} B^{\mu \nu} / 2 \\
\mathcal{O}_{\tilde{W} B}=\left(\varphi^{\dagger} \tau^{i} \varphi\right) \tilde{W}_{\mu \nu}^{i} B^{\mu \nu}, & \mathcal{O}_{W B}=\left(\varphi^{\dagger} \tau^{i} \varphi\right) W_{\mu \nu}^{i} B^{\mu \nu}
\end{array}
$$

The operators that contain the dual tensors (e.g., $\tilde{B}_{\mu \nu} \equiv \epsilon_{\mu \nu \alpha \beta} B^{\alpha \beta} / 2$ with $\epsilon_{0123}=$ +1 ) are $C P$ odd while the remaining are $C P$ even.

These operators lead to the following Feynman rules for on-shell photons, which are necessary for our later calculations:

(1) $C P$-conserving $t \bar{t} \gamma$ vertex

$$
\frac{\sqrt{2}}{\Lambda^{2}} v \alpha_{\gamma 1} k \gamma_{\mu}
$$

(2) $C P$-violating $t \bar{t} \gamma$ vertex

$$
i \frac{\sqrt{2}}{\Lambda^{2}} v \alpha_{\gamma 2} k \gamma_{\mu} \gamma_{5}
$$

\footnotetext{
${ }^{\sharp 1}$ Notice an omission of the term $i \varphi^{\dagger} \stackrel{\leftrightarrow}{D}_{\beta} \varphi / 2$ in eq.(2.14) of [16].
} 
(3) $C P$-conserving $\gamma \gamma H$ vertex

$$
-\frac{4}{\Lambda^{2}} v \alpha_{h 1}\left[\left(k_{1} k_{2}\right) g_{\mu \nu}-k_{1 \nu} k_{2 \mu}\right]
$$

(4) $C P$-violating $\gamma \gamma H$ vertex

$$
\frac{8}{\Lambda^{2}} v \alpha_{h 2} k_{1}^{\rho} k_{2}^{\sigma} \epsilon_{\rho \sigma \mu \nu}
$$

where $k$ and $k_{1,2}$ are incoming photon momenta, and $\alpha_{\gamma 1, \gamma 2, h 1, h 2}$ are defined as

$$
\begin{aligned}
& \alpha_{\gamma 1} \equiv \sin \theta_{W} \operatorname{Re}\left(\alpha_{u W}\right)+\cos \theta_{W} \operatorname{Re}\left(\alpha_{u B}^{\prime}\right), \\
& \alpha_{\gamma 2} \equiv \sin \theta_{W} \operatorname{Im}\left(\alpha_{u W}\right)+\cos \theta_{W} \operatorname{Im}\left(\alpha_{u B}^{\prime}\right), \\
& \alpha_{h 1} \equiv \sin ^{2} \theta_{W} \operatorname{Re}\left(\alpha_{\varphi W}\right)+\cos ^{2} \theta_{W} \operatorname{Re}\left(\alpha_{\varphi B}\right)-2 \sin \theta_{W} \cos \theta_{W} \operatorname{Re}\left(\alpha_{W B}\right), \\
& \alpha_{h 2} \equiv \sin ^{2} \theta_{W} \operatorname{Re}\left(\alpha_{\varphi \tilde{W}}\right)+\cos ^{2} \theta_{W} \operatorname{Re}\left(\alpha_{\varphi \tilde{B}}\right)-\sin \theta_{W} \cos \theta_{W} \operatorname{Re}\left(\alpha_{\tilde{W} B}\right)
\end{aligned}
$$

In our notation, the standard-model $f \bar{f} \gamma$ coupling is given by

$$
e Q_{f} \gamma_{\mu}
$$

where $e$ is the proton charge and $Q_{f}$ is $f$ 's electric charge in $e$ unit (e.g., $Q_{u}=2 / 3$ ).

The top-quark decay vertex is also affected by some dim.6 operators. For the onmass-shell $W$ boson it will be sufficient to consider just the following $t b W$ amplitude since other possible terms do not interfere with the SM tree-level vertex when $m_{b}$ is neglected:

$$
\Gamma_{W t b}^{\mu}=-\frac{g}{\sqrt{2}} \bar{u}\left(p_{b}\right)\left[\gamma^{\mu} f_{1}^{L} P_{L}-\frac{i \sigma^{\mu \nu} k_{\nu}}{M_{W}} f_{2}^{R} P_{R}\right] u\left(p_{t}\right)
$$

where $P_{L, R} \equiv\left(1 \mp \gamma_{5}\right) / 2$, and $f_{1}^{L}$ and $f_{2}^{R}$ are given by

$$
\begin{aligned}
& f_{1}^{L}=1+\frac{v}{\Lambda^{2}}\left[\frac{m_{t}}{2} \alpha_{D u}-2 v \alpha_{\varphi q}^{(3)}\right] \\
& f_{2}^{R}=-\frac{v}{\Lambda^{2}} M_{W}\left[\frac{4}{g} \alpha_{u W}+\frac{1}{2} \alpha_{D u}\right]
\end{aligned}
$$

with $\alpha_{D u}$ and $\alpha_{\varphi q}^{(3)}$ being the coefficients of the following operators: ${ }^{2}$.

$$
\mathcal{O}_{D u}=\left(\bar{q} D_{\mu} u\right) D^{\mu} \tilde{\varphi}, \quad \mathcal{O}_{\varphi q}^{(3)}=i\left(\varphi^{\dagger} D_{\mu} \tau^{i} \varphi\right)\left(\bar{q} \gamma^{\mu} \tau^{i} q\right)
$$

Finally, the $\nu \ell W$ vertex is assumed to receive negligible contributions from physics beyond the SM.

\footnotetext{
$\sharp 2$ Note that there is another potential source of contribution, which may come from $\mathcal{O}_{\bar{D} u}=$ $\left(D_{\mu} \bar{q}\right) u D^{\mu} \tilde{\varphi}$. However, this operator could be eliminated using equations of motion; therefore, it is neglected hereafter. We thank Ilya Ginzburg for pointing this to us.
} 


\section{Polarization asymmetries}

We are now ready to calculate the cross section of $\gamma \gamma \rightarrow t \bar{t}\left(\rightarrow \ell^{ \pm} X\right)$. The work is straightforward and we carried it out via FORM [17] as mentioned in sec.1. The analytical results are however too long to show in this paper. We therefore refrain from showing them here and we will present only some general formulas in appendix A1. As a direct application of those calculations, in this section we study $C P$-violating asymmetries. $C P$-violation phenomena would be an interesting indication of some new physics since SM contribution is negligible in the top-quark couplings.

As mentioned in the Introduction, we can form $C P$-violating asymmetries by adjusting initial-state polarizations. These are characterized by the initial electron and positron longitudinal-polarizations $P_{e}$ and $P_{\bar{e}}$, the average helicities of the initial-laser-photons $P_{\gamma}$ and $P_{\tilde{\gamma}}$, and their maximum average linear-polarizations $P_{t}$ and $P_{\tilde{t}}$ with the azimuthal angles $\varphi$ and $\tilde{\varphi}$ (defined the same way as in [4]). $P_{\gamma, t}$ and $P_{\tilde{\gamma}, \tilde{t}}$ have to satisfy

$$
0 \leq P_{\gamma}^{2}+P_{t}^{2} \leq 1, \quad 0 \leq P_{\tilde{\gamma}}^{2}+P_{\tilde{t}}^{2} \leq 1
$$

Specifically we consider the following $C P$-violating asymmetries: $\sharp 3$

$$
A_{l i n} \equiv \frac{\sigma(\chi=+\pi / 4)-\sigma(\chi=-\pi / 4)}{\sigma(\chi=+\pi / 4)+\sigma(\chi=-\pi / 4)}
$$

and

$$
A_{\text {cir }} \equiv \frac{\sigma(++)-\sigma(--)}{\sigma(++)+\sigma(--)},
$$

where $\sigma(\chi= \pm \pi / 4)$ means the total cross section of $\gamma \gamma \rightarrow t \bar{t}$ with $P_{e}=P_{\bar{e}}=1$, $P_{t}=P_{\tilde{t}}=P_{\gamma}=P_{\tilde{\gamma}}=1 / \sqrt{2}$ and $\chi \equiv \varphi-\tilde{\varphi}= \pm \pi / 4$, while $\sigma( \pm \pm)$ corresponds to the one with $P_{e}=P_{\bar{e}}=P_{\gamma}=P_{\tilde{\gamma}}= \pm 1$ (which means $P_{t}=P_{\tilde{t}}=0$ ). They were computed as functions of the Higgs-boson mass for

$$
\begin{gathered}
\sqrt{s_{e \bar{e}}}=500 \mathrm{GeV}, \quad \Lambda=1 \mathrm{TeV}, \quad y_{0}=\tilde{y}_{0}=4.828 \\
\alpha_{\gamma 2}=\alpha_{h 2}=0.1
\end{gathered}
$$

and using the SM expression for the Higgs-boson width. The results are shown in Figs 1 and 2 .

\footnotetext{
$\sharp 3$ These were used previously in [8] in order to study the $C P$ property of the Higgs boson produced in $\gamma \gamma \rightarrow H$. $A_{\text {lin }}$ was also used in [13, 14] for studying the $C P$-violating $t \bar{t} \gamma$ coupling.
} 


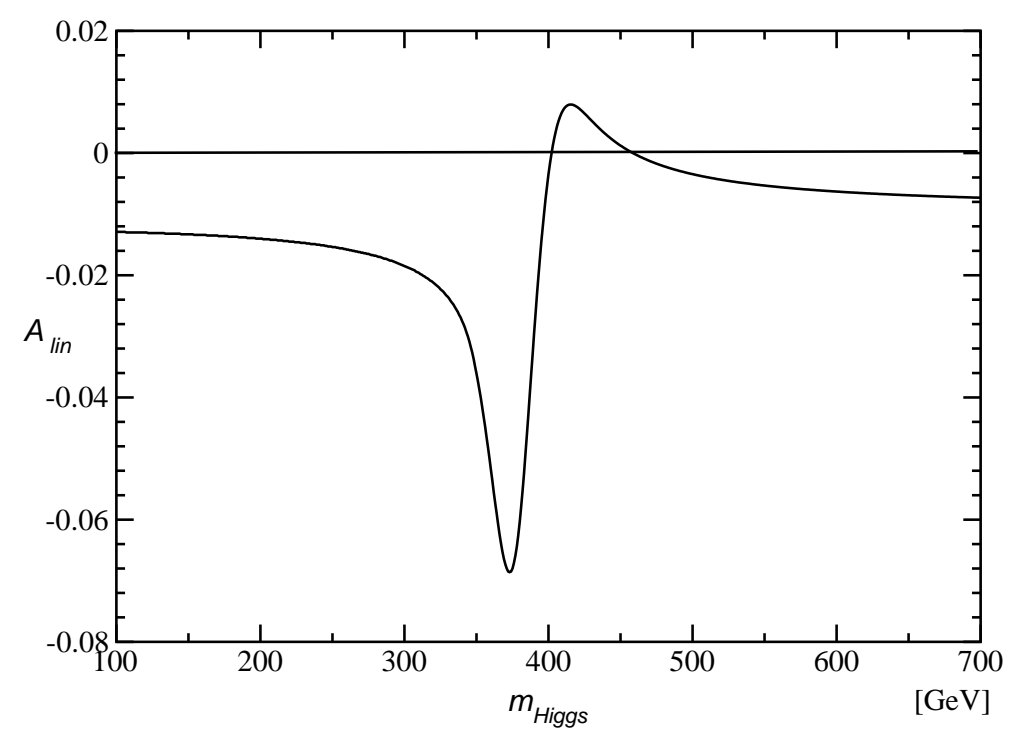

Figure 1: $C P$-violating linear-polarization asymmetry $A_{l i n}$ as a function of the Higgs-boson mass computed for $\sqrt{s_{e \bar{e}}}=500 \mathrm{GeV}, \Lambda=1 \mathrm{TeV}, y_{0}=\tilde{y}_{0}=4.828$, $\alpha_{\gamma 2}=\alpha_{h 2}=0.1, P_{e}=P_{\bar{e}}=1, P_{t}=P_{\tilde{t}}=P_{\gamma}=P_{\tilde{\gamma}}=1 / \sqrt{2}$ and $\chi \equiv \varphi-\tilde{\varphi}= \pm \pi / 4$.

The sharp peak in each asymmetry comes, of course, from the Higgs-boson pole, where the signal will be easily observed. The resonance region has been already studied in great details in existing literature [6]-[12]. Here we will study the possibility of extracting a meaningful signal when the average $\sqrt{s_{\gamma \gamma}}$ is far from the Higgs-boson mass. For the parameters adopted above one can find approximate formulas that illustrate sensitivity to $C P$-violating coefficients $\alpha_{\gamma_{2}}$ and $\alpha_{h 2}$ :

(1) $m_{H}=100 \mathrm{GeV}$

$$
\begin{aligned}
& A_{\text {lin }}=-\left(8.8 \alpha_{\gamma 2}+4.2 \alpha_{h 2}\right) \times 10^{-2}, \\
& A_{\text {cir }}=-5.2 \times 10^{-6} \alpha_{h 2} .
\end{aligned}
$$

(2) $m_{H}=300 \mathrm{GeV}$

$$
\begin{aligned}
& A_{\text {lin }}=-\left(8.8 \alpha_{\gamma 2}+11 \alpha_{h 2}\right) \times 10^{-2}, \\
& A_{\text {cir }}=-8.5 \times 10^{-2} \alpha_{h 2} .
\end{aligned}
$$

(3) $m_{H}=500 \mathrm{GeV}$

$$
A_{\text {lin }}=-\left(8.8 \alpha_{\gamma 2}-8.1 \alpha_{h 2}\right) \times 10^{-2}
$$




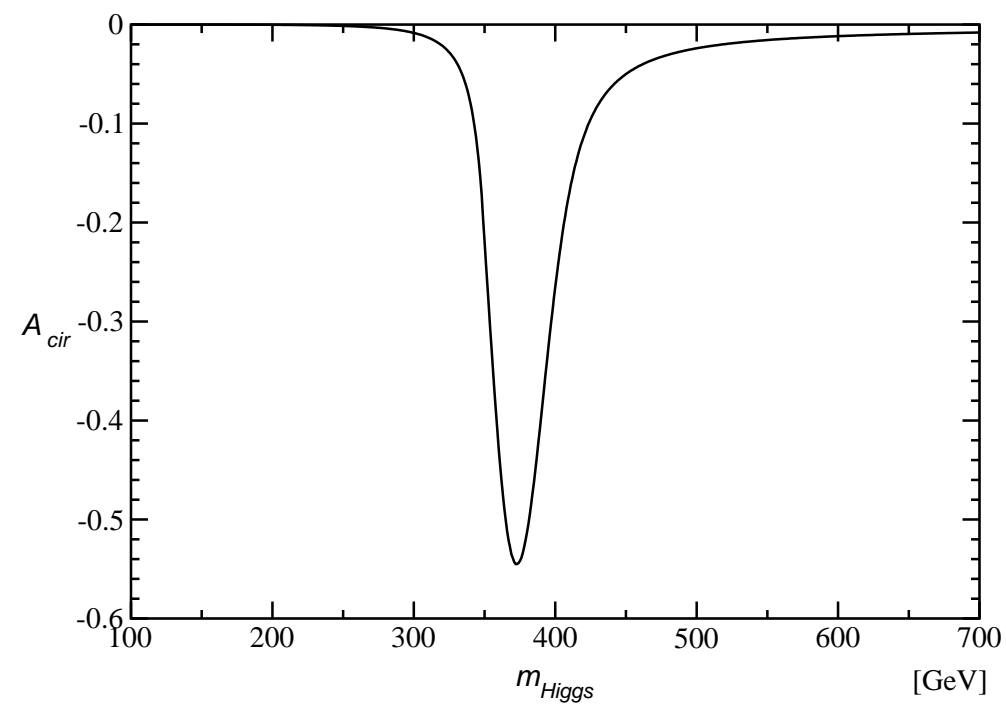

Figure 2: $C P$-violating circular-polarization asymmetry $A_{c i r}$ as a function of the Higgs-boson mass computed for $\sqrt{s_{e \bar{e}}}=500 \mathrm{GeV}, \Lambda=1 \mathrm{TeV}, y_{0}=\tilde{y}_{0}=4.828$, $\alpha_{h 2}=0.1$, and $P_{e}=P_{\bar{e}}=P_{\gamma}=P_{\tilde{\gamma}}= \pm 1$.

$$
A_{\text {cir }}=-0.24 \alpha_{h 2}
$$

Note that because of the $\gamma_{5}$ factor in the $C P$-violating $t \bar{t} \gamma$ vertex (9) its interference with the SM amplitude vanishes when the initial photons have pure circular polarizations [13. This is why the $\alpha_{\gamma 2}$ contribution to $A_{c i r}$ is zero.

Since the asymmetries are all small, their expected statistical errors in actual measurements can be computed by

$$
\Delta A_{\text {lin, }, \mathrm{ir}} \simeq 1 / \sqrt{N_{t \bar{t}}}
$$

and consequently their statistical significances are estimated as

$$
N_{S D} \equiv|A| / \Delta A \simeq|A| \sqrt{N_{t \bar{t}}}
$$

We estimate $N_{t \bar{t}} \sim 36,000$ and 21,000 events for the linear and circular polarizations respectively assuming that a luminosity of $L_{e \bar{e}}^{\text {eff }} \equiv \epsilon L_{e \bar{e}}=500 \mathrm{fb}^{-1}$ can be reached for each mode ( $\epsilon$ denotes the relevant detection efficiency and $L_{e \bar{e}}$ is the integrated luminosity). In this case deviations from the SM will be observable provided the coefficients $\alpha_{\gamma 2}$ and $\alpha_{h 2}$ are of the order of 0.1 . 


\section{Optimal-observable analysis}

Let us briefly summarize the main points of this method first. Suppose we have a differential cross section of the following form

$$
\frac{d \sigma}{d \phi}=f_{0}(\phi)+\sum_{i} c_{i} f_{i}(\phi),
$$

where $f_{0}(\phi)$ is the SM differential cross section, while $f_{i}(\phi)$ 's are known functions (of the same order of magnitude as $f_{0}(\phi)$ ) of the location in final-state phase-space $\phi$, and $c_{i}$ are model-dependent dimensionless coefficients expressing non-standard contributions. We assume that $c_{i}$ are so small that we can safely drop $\mathcal{O}\left(c_{i}^{2}\right)$ contributions. The goal would be to determine $c_{i}$ 's. It can be done by using appropriate weighting-functions $w_{i}(\phi)$ such that

$$
c_{i}=\left[\int d \phi w_{i}(\phi) \frac{d \sigma}{d \phi}\right] / \int d \phi f_{0}(\phi) .
$$

(Note here that $d \sigma / d \phi$ is not always a properly normalized probability distribution.) Generally, different choices for $w_{i}(\phi)$ are possible, but there is a unique choice which minimizes the resultant statistical error. Such weighting functions are given by [21]

$$
w_{i}(\phi)=I_{0} \sum_{j} X_{i j}\left[f_{j}(\phi) / f_{0}(\phi)-I_{j} / I_{0}\right],
$$

where $X_{i j}$ is the inverse matrix of $M_{i j}$ defined as $\sharp^{\sharp}$

$$
M_{i j} \equiv \int d \phi f_{i}(\phi) f_{j}(\phi) / f_{0}(\phi)-I_{i} I_{j} / I_{0},
$$

and

$$
I_{0} \equiv \int d \phi f_{0}(\phi), \quad I_{i} \equiv \int d \phi f_{i}(\phi) .
$$

For the weighting functions chosen in the above manner, the covariance matrix $V_{i j}$ that corresponds to $w_{i}(\phi)$ becomes

$$
V_{i j}=I_{0} X_{i j} / N+\mathcal{O}\left(c_{i}\right),
$$

\footnotetext{
${ }^{\sharp 4}$ If we made the corresponding matrix including the SM term, it would become $\mathcal{M}_{i j}=$ $\int d \phi f_{i}(\phi) f_{j}(\phi) / f_{0}(\phi)$, where the zero-th component is the SM contribution. This matrix is what we have used in our previous papers. It is a simple linear-algebra exercise to show that this $\mathcal{M}_{i j}$ and $M_{i j}$ in eq.(32) both give the same $X_{i j}$ when restricted to the non-SM components.
} 
where $N$ is the total number of collected events. Its diagonal elements give expected statistical uncertainty for measurements of $c_{i}$ as

$$
\left|\Delta c_{i}\right|=\sqrt{V_{i i}}
$$

We are going to apply this technique to the final-lepton angular and energy distribution of $\gamma \gamma \rightarrow t \bar{t} \rightarrow \ell^{+} X$

$$
\begin{gathered}
\frac{d \sigma}{d E_{\ell} d \cos \theta_{\ell}}=f_{\mathrm{SM}}\left(E_{\ell}, \cos \theta_{\ell}\right)+\alpha_{\gamma 1} f_{\gamma 1}\left(E_{\ell}, \cos \theta_{\ell}\right)+\alpha_{\gamma 2} f_{\gamma 2}\left(E_{\ell}, \cos \theta_{\ell}\right) \\
+\alpha_{h 1} f_{h 1}\left(E_{\ell}, \cos \theta_{\ell}\right)+\alpha_{h 2} f_{h 2}\left(E_{\ell}, \cos \theta_{\ell}\right)+\alpha_{d} f_{d}\left(E_{\ell}, \cos \theta_{\ell}\right)
\end{gathered}
$$

in the $e \bar{e}$-CM frame. Here $f_{\mathrm{SM}}$ denotes the standard-model contribution, $f_{\gamma 1, \gamma 2}$ describe, respectively, the anomalous $C P$-conserving- and $C P$-violating- $t \bar{t} \gamma$-vertices contributions, $f_{h 1, h 2}$ those generated by the anomalous $C P$-conserving and $C P$ violating $\gamma \gamma H$-vertices, and $f_{d}$ that by the anomalous $t b W$-vertex with

$$
\alpha_{d}=\operatorname{Re}\left(f_{2}^{R}\right)
$$

See the Appendix for details of our calculation framework.

The covariance matrix $V_{i j} \propto X_{i j}$ determines our ability to measure the coefficients $c_{i}$. However in the case considered here, it turns out that our results for $V_{i j}$ are very unstable: even a tiny fluctuation of $M_{i j}$ changes $X_{i j}$ significantly. This indicates that some of $f_{i}$ have similar shapes ${ }^{5}$ and therefore their coefficients cannot be easily disentangled. The only option in such a case is to refrain from determining all the couplings at once through this process alone. Therefore hereafter we assume that some of $c_{i}$ 's have been measured in other processes (e.g. in $\left.e \bar{e} \rightarrow t \bar{t} \rightarrow \ell^{ \pm} X\right)$.

In order to carry out these studies numerically, we first give full elements of

$$
\mathcal{M}_{I J}=\int d E_{\ell} d \cos \theta_{\ell} f_{I}\left(E_{\ell}, \cos \theta_{\ell}\right) f_{J}\left(E_{\ell}, \cos \theta_{\ell}\right) / f_{1}\left(E_{\ell}, \cos \theta_{\ell}\right)
$$

where $I, J=1, \cdots, 6$ correspond to SM, $\gamma 1, \gamma 2, h 1, h 2$ and $d$ respectively as our basis. The $(i, j)$ elements $(i, j=2 \sim 6)$ of $\mathcal{M}$ 's inverse matrix coincide with $X_{i j}$ appearing in eq.(31) as mentioned in footnote 3.

\footnotetext{
${ }^{\sharp 5}$ Note that if two $f_{i}$ functions were proportional to each other then the matrix $M_{i j}$ would have a vanishing determinant, and therefore its inverse $X_{i j}$ could not be determined.
} 
(1) Linear Polarization

We took $P_{e}=P_{\bar{e}}=1, P_{t}=P_{\tilde{t}}=P_{\gamma}=P_{\tilde{\gamma}}=1 / \sqrt{2}$ and $\chi=\pi / 4$, which were used to compute $A_{\text {lin }}$ in the previous section as typical linear-polarization parameters.

1-1) $m_{H}=100 \mathrm{GeV}$

$$
\begin{aligned}
& \mathcal{M}_{11}=0.810 \cdot 10^{1}, \quad \mathcal{M}_{12}=0.173 \cdot 10^{2}, \quad \mathcal{M}_{13}=-0.703, \\
& \mathcal{M}_{14}=-0.319 \cdot 10^{1}, \quad \mathcal{M}_{15}=-0.337, \quad \mathcal{M}_{16}=0 \text {, } \\
& \mathcal{M}_{22}=0.377 \cdot 10^{2}, \quad \mathcal{M}_{23}=-0.153 \cdot 10^{1}, \quad \mathcal{M}_{24}=-0.627 \cdot 10^{1} \text {, } \\
& \mathcal{M}_{25}=-0.730, \quad \mathcal{M}_{26}=0.125 \cdot 10^{1}, \quad \mathcal{M}_{33}=0.638 \cdot 10^{-1} \text {, } \\
& \mathcal{M}_{34}=0.248, \quad \mathcal{M}_{35}=0.305 \cdot 10^{-1}, \quad \mathcal{M}_{36}=-0.683 \cdot 10^{-1} \text {, } \\
& \mathcal{M}_{44}=0.183 \cdot 10^{1}, \quad \mathcal{M}_{45}=0.122, \quad \mathcal{M}_{46}=0.135 \cdot 10^{1} \text {, } \\
& \mathcal{M}_{55}=0.149 \cdot 10^{-1}, \quad \mathcal{M}_{56}=-0.263 \cdot 10^{-1}, \quad \mathcal{M}_{66}=0.392 \cdot 10^{1} \text {. }
\end{aligned}
$$

1-2) $m_{H}=300 \mathrm{GeV}$

$$
\begin{aligned}
& \mathcal{M}_{11}=0.810 \cdot 10^{1}, \quad \mathcal{M}_{12}=0.173 \cdot 10^{2}, \quad \mathcal{M}_{13}=-0.703, \\
& \mathcal{M}_{14}=-0.790 \cdot 10^{1}, \quad \mathcal{M}_{15}=-0.152 \cdot 10^{1}, \quad \mathcal{M}_{16}=0, \\
& \mathcal{M}_{22}=0.377 \cdot 10^{2}, \quad \mathcal{M}_{23}=-0.153 \cdot 10^{1}, \quad \mathcal{M}_{24}=-0.156 \cdot 10^{2}, \\
& \mathcal{M}_{25}=-0.314 \cdot 10^{1}, \quad \mathcal{M}_{26}=0.125 \cdot 10^{1}, \quad \mathcal{M}_{33}=0.638 \cdot 10^{-1} \text {, } \\
& \mathcal{M}_{34}=0.616, \quad \mathcal{M}_{35}=0.128, \quad \mathcal{M}_{36}=-0.683 \cdot 10^{-1} \text {, } \\
& \mathcal{M}_{44}=0.112 \cdot 10^{2}, \quad \mathcal{M}_{45}=0.176 \cdot 10^{1}, \quad \mathcal{M}_{46}=0.332 \cdot 10^{1}, \\
& \mathcal{M}_{55}=0.309, \quad \mathcal{M}_{56}=0.260, \quad \mathcal{M}_{66}=0.392 \cdot 10^{1} .
\end{aligned}
$$

1-3) $m_{H}=500 \mathrm{GeV}$

$$
\begin{array}{lll}
\mathcal{M}_{11}=0.810 \cdot 10^{1}, & \mathcal{M}_{12}=0.173 \cdot 10^{2}, & \mathcal{M}_{13}=-0.703 \\
\mathcal{M}_{14}=0.374 \cdot 10^{1}, & \mathcal{M}_{15}=-0.221 \cdot 10^{1}, & \mathcal{M}_{16}=0 \\
\mathcal{M}_{22}=0.377 \cdot 10^{2}, & \mathcal{M}_{23}=-0.153 \cdot 10^{1}, & \mathcal{M}_{24}=0.737 \cdot 10^{1} \\
\mathcal{M}_{25}=-0.425 \cdot 10^{1}, & \mathcal{M}_{26}=0.125 \cdot 10^{1}, & \mathcal{M}_{33}=0.638 \cdot 10^{-1} \\
\mathcal{M}_{34}=-0.293, & \mathcal{M}_{35}=0.166, & \\
\mathcal{M}_{44}=0.251 \cdot 10^{1}, & \mathcal{M}_{45}=-0.165 \cdot 10^{1}, & \mathcal{M}_{46}=-0.683 \cdot 10^{-1} \\
\mathcal{M}_{55}=0.110 \cdot 10^{1}, & \mathcal{M}_{56}=0.122 \cdot 10^{1}, & \mathcal{M}_{66}=0.392 \cdot 10^{1}
\end{array}
$$

(2) Circular Polarization

What we took as circular-polarization parameters are also those used for $A_{\text {cir }}$ : $P_{e}=P_{\bar{e}}=P_{\gamma}=P_{\tilde{\gamma}}=1$.

2-1) $m_{H}=100 \mathrm{GeV}$

$$
\begin{array}{lll}
\mathcal{M}_{11}=0.460 \cdot 10^{1}, & \mathcal{M}_{12}=0.996 \cdot 10^{1}, & \mathcal{M}_{13}=0 \\
\mathcal{M}_{14}=-0.152 \cdot 10^{1}, & \mathcal{M}_{15}=-0.240 \cdot 10^{-4}, & \mathcal{M}_{16}=0 \\
\mathcal{M}_{22}=0.219 \cdot 10^{2}, & \mathcal{M}_{23}=0, & \mathcal{M}_{24}=-0.306 \cdot 10^{1}, \\
\mathcal{M}_{25}=-0.481 \cdot 10^{-4}, & \mathcal{M}_{26}=0.611, & \mathcal{M}_{33}=0 \\
\mathcal{M}_{34}=0, & \mathcal{M}_{35}=0, & \mathcal{M}_{36}=0, \\
\mathcal{M}_{44}=0.748, & \mathcal{M}_{45}=0.122 \cdot 10^{-4}, & \mathcal{M}_{46}=0.660, \\
\mathcal{M}_{55}=0.198 \cdot 10^{-9}, & \mathcal{M}_{56}=0.114 \cdot 10^{-4}, & \mathcal{M}_{66}=0.229 \cdot 10^{1}
\end{array}
$$


2-2) $m_{H}=300 \mathrm{GeV}$

$$
\begin{array}{lll}
\mathcal{M}_{11}=0.460 \cdot 10^{1}, & \mathcal{M}_{12}=0.996 \cdot 10^{1}, & \mathcal{M}_{13}=0, \\
\mathcal{M}_{14}=-0.392 \cdot 10^{1}, & \mathcal{M}_{15}=-0.389, & \mathcal{M}_{16}=0, \\
\mathcal{M}_{22}=0.219 \cdot 10^{2}, & \mathcal{M}_{23}=0, & \mathcal{M}_{24}=-0.793 \cdot 10^{1}, \\
\mathcal{M}_{25}=-0.781, & \mathcal{M}_{26}=0.611, & \mathcal{M}_{33}=0, \\
\mathcal{M}_{34}=0, & \mathcal{M}_{35}=0, & \mathcal{M}_{36}=0, \\
\mathcal{M}_{44}=0.497 \cdot 10^{1}, & \mathcal{M}_{45}=0.502, & \mathcal{M}_{46}=0.171 \cdot 10^{1}, \\
\mathcal{M}_{55}=0.511 \cdot 10^{-1}, & \mathcal{M}_{56}=0.181, & \mathcal{M}_{66}=0.229 \cdot 10^{1} .
\end{array}
$$

2-3) $m_{H}=500 \mathrm{GeV}$

$$
\begin{array}{llll}
\mathcal{M}_{11}=0.460 \cdot 10^{1}, & \mathcal{M}_{12}=0.996 \cdot 10^{1}, & \mathcal{M}_{13}=0, \\
\mathcal{M}_{14}=0.168 \cdot 10^{1}, & \mathcal{M}_{15}=-0.110 \cdot 10^{1}, & \mathcal{M}_{16}=0, \\
\mathcal{M}_{22}=0.219 \cdot 10^{2}, & \mathcal{M}_{23}=0, & & \mathcal{M}_{24}=0.338 \cdot 10^{1}, \\
\mathcal{M}_{25}=-0.221 \cdot 10^{1}, & \mathcal{M}_{26}=0.611, & \mathcal{M}_{33}=0, \\
\mathcal{M}_{34}=0, & \mathcal{M}_{35}=0, & \mathcal{M}_{36}=0, \\
\mathcal{M}_{44}=0.920, & \mathcal{M}_{45}=-0.634, & \mathcal{M}_{46}=-0.728, \\
\mathcal{M}_{55}=0.436, & \mathcal{M}_{56}=0.537, & \mathcal{M}_{66}=0.229 \cdot 10^{1} .
\end{array}
$$

All the elements $\mathcal{M}_{i j}$ above are given in units of fb. In these results, the third components of $\mathcal{M}$ for the circular polarization vanish as was mentioned in sec.4. Also, in accordance with the decoupling theorem shown in [22], the elements $\mathcal{M}_{16}$ are always zero within our approximation of neglecting contributions quadratic in non-standard interactions and treating the decaying $t$ and $W$ as on-shell particles.

When estimating the statistical uncertainty in simultaneous measurements of, e.g., $\alpha_{\gamma 1}$ and $\alpha_{h 1}$ (assuming all other coefficients are known), we need only the components with indices 1,2 and 4. Let us express the resultant uncertainties as $\Delta \alpha_{\gamma 1}^{[3]}$ and $\Delta \alpha_{h 1}^{[3]}$, where "3" means that we took account of the input $\mathcal{M}_{i j}$ up to three decimal places. In order to see how stable the results are, we also computed $\Delta \alpha_{\gamma 1}^{[2]}$ and $\Delta \alpha_{h 1}^{[2]}$ by rounding $\mathcal{M}_{i j}$ off to two decimal places. Then, if both of the deviations $\left|\Delta \alpha_{\gamma 1, h 1}^{[3]}-\Delta \alpha_{\gamma 1, h 1}^{[2]}\right| / \Delta \alpha_{\gamma 1, h 1}^{[3]}$ are less than $10 \%$, we adopted the results as stable solutions. Therefore, the ambiguity of the following results $\Delta \alpha_{i}$, which are $\Delta \alpha_{i}^{[3]}$, is at most $10 \%$.

1) Linear Polarization

What we obtained as stable solutions for linear polarization are

- Independent of $m_{H}$

$$
\Delta \alpha_{\gamma 2}=73 / \sqrt{N_{\ell}}, \quad \Delta \alpha_{d}=1.9 / \sqrt{N_{\ell}}
$$


- $m_{H}=100 \mathrm{GeV}$

$$
\Delta \alpha_{h 2}=107 / \sqrt{N_{\ell}}, \quad \Delta \alpha_{d}=1.6 / \sqrt{N_{\ell}}
$$

- $m_{H}=300 \mathrm{GeV}$

$$
\Delta \alpha_{h 1}=3.4 / \sqrt{N_{\ell}}, \quad \Delta \alpha_{d}=3.2 / \sqrt{N_{\ell}}
$$

where $N_{\ell} \simeq 4,000$ for $L_{e \bar{e}}^{\mathrm{eff}}=500 \mathrm{fb}^{-1}$.

2) Circular Polarization

Stable solutions which we found for circular polarization are

- $m_{H}=100 \mathrm{GeV}$

$$
\Delta \alpha_{h 1}=9.0 / \sqrt{N_{\ell}}, \quad \Delta \alpha_{d}=3.0 / \sqrt{N_{\ell}}
$$

- $m_{H}=300 \mathrm{GeV}$

$$
\begin{array}{ll}
\Delta \alpha_{h 1}=3.5 / \sqrt{N_{\ell}}, & \Delta \alpha_{d}=3.0 / \sqrt{N_{\ell}}, \\
\Delta \alpha_{h 2}=35 / \sqrt{N_{\ell}}, & \Delta \alpha_{d}=3.1 / \sqrt{N_{\ell}},
\end{array}
$$

- $m_{H}=500 \mathrm{GeV}$

$$
\begin{array}{ll}
\Delta \alpha_{h 1}=7.7 / \sqrt{N_{\ell}}, & \Delta \alpha_{d}=2.8 / \sqrt{N_{\ell}}, \\
\Delta \alpha_{h 2}=10 / \sqrt{N_{\ell}}, & \Delta \alpha_{d}=2.8 / \sqrt{N_{\ell}}
\end{array}
$$

where $N_{\ell} \simeq 2,300$ for $L_{e \bar{e}}^{\mathrm{eff}}=500 \mathrm{fb}^{-1} \sharp 6$

Unfortunately, we did not find any stable solution including $\Delta \alpha_{\gamma 1}$. We therefore have to look for other suitable processes to determine this parameter. The precision of $\alpha_{\gamma 2}$ is not satisfactory either, but since this is a $C P$-violating parameter, we will be able to get some information on it from the $C P$ asymmetries which we gave in the previous section.

The above results are for $\Lambda=1 \mathrm{TeV}$. When one takes the new-physics scale to be $\Lambda^{\prime}=\lambda \Lambda$, then all the above results $\left(\Delta \alpha_{i}\right)$ are replaced with $\Delta \alpha_{i} / \lambda^{2}$, which means that the right-hand sides of eqs.(42)-(49) are multiplied by $\lambda^{2}$.

\footnotetext{
${ }^{\sharp 6}$ We used the tree-level SM formula for computing $N_{\ell}$, so we have the same $N_{\ell}$ for different $m_{H}$.
} 
It should be mentioned here that the above estimation of expected errors does not take into account a possible background. However the top-quark tagging in the one-lepton and six-jets final state is relatively straightforward and therefore should not increase the uncertainties found above very dramatically. Of course, in the real experiment, one will have to redo the analysis taking into account not only the background, but also all the systematic errors which are unknown at present. Therefore the fully realistic analysis cannot be performed at this moment.

\section{Summary and discussion}

We have studied here beyond-the-SM effects in the process $\gamma \gamma \rightarrow t \bar{t}\left(\rightarrow \ell^{+} X\right)$ for arbitrarily-polarized photon beams, taking advantage of the fact that one can control polarizations of the incoming photon beams. Non-standard interactions have been parameterized through dim.6 local and gauge symmetric effective operators à la Buchmüller and Wyler [16 toward the first realistic comprehensive modelindependent analysis of the process. We listed all the necessary operators and the corresponding Feynman rules. It was shown that in the list of operators that seem to contribute there are some which are redundant and should be dropped in the analysis. Assuming that those new-physics (NP) effects are small, we have kept only terms linear in modification of the SM tree vertices.

We first computed two $C P$-violating polarization asymmetries for linear and circular photon polarizations, and found there are good chances of detecting their signals. We then applied the optimal-observable technique to the final-leptonmomentum distribution, and estimated statistical significances of measuring each NP-parameters. Unfortunately, we had to conclude that it is never realistic to try to determine all the independent NP-parameters at once through $\gamma \gamma \rightarrow t \bar{t} \rightarrow \ell^{ \pm} X$ alone, but still we would be able to perform useful analysis if we could utilize complementary information collected in other independent processes.

We have not discussed here a possible background. This is mainly because identifying the $t \bar{t}$ final state in semileptonic-hadronic decays is easy, since we always have a very energetic charged lepton and the other (anti)top quark decaying purely hadronically with an invariant mass of $m_{t}$. That is, the tagging in this particular final state will be relatively easy. However, some comments on background esti- 
mation will be helpful. Although the most serious background is $W$-boson pair productions and indeed its total cross section could be larger than $\sigma_{\text {tot }}(t \bar{t})$, a dedicated simulation study [23] has shown that $t \bar{t}$ events can be selected with signalto-background ratio of 10 by imposing appropriate invariant-mass constraints on the final-particle momenta.

It should be stressed that for the purpose of future data analysis it is unavoidable to have a tool which would allow consistently to control all possible effects. For instance it is conceivable that some non-standard effects from the top-quark decays would mimic another non-standard interactions from the $t \bar{t}$ production process, therefore without an analysis that allows to control all those contribution any meaningful data analysis would be impossible.

Finally, it should be emphasized here that the effective-operator strategy adopted in this article is valid only for $\Lambda \gg v \sim 250 \mathrm{GeV}$, in contrast with $e^{+} e^{-} \rightarrow t \bar{t} \rightarrow$ $\ell^{ \pm} X$. Should the reaction $\gamma \gamma \rightarrow t \bar{t} \rightarrow \ell^{ \pm} X$ exhibit a deviation from the SM predictions that cannot be described properly within this framework, this would be an indication of a low-energy beyond-the-SM physics, e.g., two Higgs-doublet model with relatively low mass scale of new scalar degrees of freedom.

\section{ACKNOWLEDGMENTS}

One of us (Z.H.) would like to thank Tohru Takahashi for very useful discussion, Isamu Watanabe for showing some results of their numerical computations, and Eri Asakawa and Saurabh Rindani for kind correspondence on their works. B.G. thanks Mikolaj Misiak for useful discussions concerning effective Lagrangians. This work is supported in part by the State Committee for Scientific Research (Poland) under grant 1 P03B 07826 in the period 2004-2006 and the Grant-in-Aid for Scientific Research No.13135219 from the Japan Society for the Promotion of Science.

\section{APPENDIX}

A1. Cross section for $\gamma \gamma \rightarrow t \bar{t} \rightarrow \ell^{ \pm} X$ 
For photons with definite polarizations the cross section for $\gamma \gamma \rightarrow t \bar{t}$ is given by

$$
\begin{aligned}
d \sigma(\gamma \gamma & \rightarrow t \bar{t})=C\left|\mathcal{M}_{\alpha \beta} \epsilon^{\alpha}\left(h_{1}\right) \tilde{\epsilon}^{\beta}\left(h_{2}\right)\right|^{2} \\
& =C \mathcal{M}_{\alpha \beta} \epsilon^{\alpha}\left(h_{1}\right) \tilde{\epsilon}^{\beta}\left(h_{2}\right) \mathcal{M}_{\rho \sigma}^{*} \epsilon^{* \rho}\left(h_{1}\right) \tilde{\epsilon}^{* \sigma}\left(h_{2}\right)
\end{aligned}
$$

where $C$ expresses the kinematically-determined part, and $h_{1,2}$ are the helicities of the initial two photons:

$$
\epsilon^{\mu}\left(h_{1}\right)=\frac{1}{\sqrt{2}}\left(\epsilon_{1}^{\mu}+i h_{1} \epsilon_{2}^{\mu}\right), \quad \tilde{\epsilon}^{\mu}\left(h_{2}\right)=\frac{1}{\sqrt{2}}\left(-\epsilon_{1}^{\mu}+i h_{2} \epsilon_{2}^{\mu}\right)
$$

with $\epsilon_{1}^{\mu}=(0,1,0,0)$ and $\epsilon_{2}^{\mu}=(0,0,1,0)$. Since $\epsilon^{\mu}( \pm 1)$ and $\tilde{\epsilon}^{\mu}( \pm 1)$ are

$$
\epsilon^{\mu}( \pm 1)=\frac{1}{\sqrt{2}}\left(\epsilon_{1}^{\mu} \pm i \epsilon_{2}^{\mu}\right), \quad \tilde{\epsilon}^{\mu}( \pm 1)=\frac{1}{\sqrt{2}}\left(-\epsilon_{1}^{\mu} \pm i \epsilon_{2}^{\mu}\right)
$$

we can express $\epsilon^{\mu}\left(h_{1}\right)$ and $\tilde{\epsilon}^{\mu}\left(h_{2}\right)$ as

$$
\epsilon^{\mu}\left(h_{1}\right)=\sum_{a= \pm 1} e^{a} \epsilon^{\mu}(a), \quad \tilde{\epsilon}^{\mu}\left(h_{2}\right)=\sum_{a= \pm 1} \tilde{e}^{a} \tilde{\epsilon}^{\mu}(a)
$$

where the coefficients are $e^{ \pm 1}=\left(1 \pm h_{1}\right) / 2$ and $\tilde{e}^{ \pm 1}=\left(1 \pm h_{2}\right) / 2$. In terms of these quantities, the above cross section is

$$
\begin{aligned}
d \sigma(\gamma \gamma \rightarrow t \bar{t}) & =C \sum_{a, b, c, d= \pm 1} e^{a} e^{c *} \tilde{e}^{b} \tilde{e}^{d *} \mathcal{M}_{\alpha \beta} \mathcal{M}_{\rho \sigma}^{*} \epsilon^{\alpha}(a) \epsilon^{* \rho}(c) \tilde{\epsilon}^{\beta}(b) \tilde{\epsilon}^{* \sigma}(d) \\
& \equiv C \sum_{a, b, c, d= \pm 1} e^{a} e^{c *} \tilde{e}^{b} \tilde{e}^{d *} \mathcal{T}^{a c, b d}
\end{aligned}
$$

The actual cross section $d \sigma(\gamma \gamma \rightarrow t \bar{t})$ measured at experiments is obtained by multiplying the above cross section with the photon-spectra functions $d N / d y$ and $d N / d \tilde{y}$, which work similarly to the parton-distribution functions inside hadrons and replacing $e^{a} e^{c *}$ and $\tilde{e}^{b} \tilde{e}^{d *}$ with the spin density matrices $\rho$ and $\tilde{\rho}$ respectively,

$$
d \sigma=\sum_{a, b, c, d= \pm 1} \int d y d \tilde{y} \frac{d N(y)}{d y} \frac{d N(\tilde{y})}{d \tilde{y}} \rho^{a c}(y) \tilde{\rho}^{b d}(\tilde{y}) d \sigma^{a c, b d}(y, \tilde{y}),
$$

where

$$
d \sigma^{a c, b d}(y, \tilde{y})=C \mathcal{T}^{a c, b d}(y, \tilde{y})
$$

is the cross section for two initial photons carrying energy-fraction $y$ and $\tilde{y}$ of those of $e$ and $\bar{e}, \rho(y)$ and $\tilde{\rho}(\tilde{y})$ are expressed in terms of the three Stokes parameters as

$$
\rho(y)=\frac{1}{2}\left(\begin{array}{cc}
1+\xi_{2}(y) & \xi_{3}(y)-i \xi_{1}(y) \\
\xi_{3}(y)+i \xi_{1}(y) & 1-\xi_{2}(y)
\end{array}\right),
$$




$$
\tilde{\rho}(\tilde{y})=\frac{1}{2}\left(\begin{array}{cc}
1+\tilde{\xi}_{2}(\tilde{y}) & \tilde{\xi}_{3}(\tilde{y})+i \tilde{\xi}_{1}(\tilde{y}) \\
\tilde{\xi}_{3}(\tilde{y})-i \tilde{\xi}_{1}(\tilde{y}) & 1-\tilde{\xi}_{2}(\tilde{y})
\end{array}\right)
$$

in our choice of polarization vectors (511). Since $d \sigma^{a c, b d}(y, \tilde{y})$ is Lorentz-invariant, we can calculate it in the $t \bar{t}(\gamma \gamma)$-CM frame.

The maximum of $y$ and $\tilde{y}$ is given by

$$
y_{\max }=\tilde{y}_{\max }=y_{0} /\left(1+y_{0}\right) \text { and } 0 \leq y_{0} \leq 2(1+\sqrt{2}) \simeq 4.828 \text {. }
$$

Since we are interested in $t \bar{t}$ productions, $y$ and $\tilde{y}$ must satisfy

$$
y \tilde{y} \geq 4 m_{t}^{2} / s .
$$

Therefore the upper and lower bounds on these variables in the integrations of eq.(54) are

$$
\begin{array}{ll}
y_{\max }=y_{0} /\left(1+y_{0}\right), & y_{\min }=4 m_{t}^{2} /\left(s \tilde{y}_{\max }\right), \\
\tilde{y}_{\max }=y_{0} /\left(1+y_{0}\right), & \tilde{y}_{\text {min }}=4 m_{t}^{2} /(s y) .
\end{array}
$$

The photon-spectrum function $d N(y) / d y$ and $\xi_{i}(y)$ in the spin density matrix $\rho(y)$ immediately after its production at the conversion point are given by the following formulas:

$$
\begin{aligned}
& d N(y) / d y=C(y) / D\left(y_{0}\right), \\
& \xi_{1}(y)=2 P_{t} \sin (2 \varphi)[r(y)]^{2} / C(y), \\
& \xi_{2}(y)=\left[P_{e} f_{2}(y)+P_{\gamma} f_{3}(y)\right] / C(y), \\
& \xi_{3}(y)=2 P_{t} \cos (2 \varphi)[r(y)]^{2} / C(y),
\end{aligned}
$$

where

$$
\begin{aligned}
& C(y)=f_{0}(y)+P_{e} P_{\gamma} f_{1}(y), \\
& D\left(y_{0}\right)=D_{0}\left(y_{0}\right)+P_{e} P_{\gamma} D_{1}\left(y_{0}\right), \\
& f_{0}(y)=1 /(1-y)+1-y-4 r(y)[1-r(y)], \\
& f_{1}(y)=y(2-y)[1-2 r(y)] /(1-y), \\
& f_{2}(y)=y_{0} r(y)\left[1+(1-y)[1-2 r(y)]^{2}\right], \\
& f_{3}(y)=[1-2 r(y)][1 /(1-y)+1-y],
\end{aligned}
$$




$$
\begin{aligned}
r(y)=y /\left[y_{0}(1-y)\right] & \\
D_{0}\left(y_{0}\right)= & \left(1-4 / y_{0}-8 / y_{0}^{2}\right) \ln \left(1+y_{0}\right)+1 / 2 \\
& +8 / y_{0}-1 /\left[2\left(1+y_{0}\right)^{2}\right] \\
D_{1}\left(y_{0}\right)= & \left(1+2 / y_{0}\right) \ln \left(1+y_{0}\right)-5 / 2+1 /\left(1+y_{0}\right) \\
& -1 /\left[2\left(1+y_{0}\right)^{2}\right]
\end{aligned}
$$

which are of course common to $d N(\tilde{y}) / d \tilde{y}$ and $\tilde{\xi}_{i}(\tilde{y})$ too.

Finally, combining thus-calculated $d \sigma(\gamma \gamma \rightarrow t \bar{t})$ with $d \Gamma(t \rightarrow \ell X)$ through the Kawasaki-Shirafuji-Tsai formula [24, we arrive at the cross section $d \sigma(\gamma \gamma \rightarrow t \bar{t} \rightarrow$ $\left.\ell^{ \pm} X\right)$

$$
\begin{aligned}
\frac{d \sigma}{d \tilde{\boldsymbol{p}}_{\ell}} & \equiv \frac{d \sigma}{d \tilde{\boldsymbol{p}}_{\ell}}(1+2 \rightarrow t+\cdots \rightarrow \ell+\cdots) \\
& =2 \int d \tilde{\boldsymbol{p}}_{t} \frac{d \sigma}{d \tilde{\boldsymbol{p}}_{t}}\left(s_{t}=n\right) \frac{1}{\Gamma_{t}} \frac{d \Gamma}{d \tilde{\boldsymbol{p}}_{\ell}}=2 B_{\ell} \int d \tilde{\boldsymbol{p}}_{t} \frac{d \sigma}{d \tilde{\boldsymbol{p}}_{t}}\left(s_{t}=n\right) \frac{1}{\Gamma} \frac{d \Gamma}{d \tilde{\boldsymbol{p}}_{\ell}}
\end{aligned}
$$

Here $d \tilde{\boldsymbol{p}}$ denotes the Lorentz-invariant phase-space element $d \boldsymbol{p} /\left[(2 \pi)^{3} 2 p^{0}\right], d \Gamma / d \tilde{\boldsymbol{p}}_{\ell}$ is the spin-averaged top-quark width

$$
\frac{d \Gamma}{d \tilde{\boldsymbol{p}}_{\ell}} \equiv \frac{d \Gamma}{d \tilde{\boldsymbol{p}}_{\ell}}(t \rightarrow \ell+\cdots),
$$

$B_{\ell} \equiv \Gamma / \Gamma_{t}$, and $d \sigma\left(s_{t}=n\right) / d \tilde{\boldsymbol{p}}_{t}$ is the single-top-quark inclusive cross section

$$
\frac{d \sigma}{d \tilde{\boldsymbol{p}}_{t}}\left(s_{t}=n\right) \equiv \frac{d \sigma}{d \tilde{\boldsymbol{p}}_{t}}\left(1+2 \rightarrow t+\cdots ; s_{t}=n\right)
$$

with the polarization vector $s_{t}$ being replaced with the so-called "effective polarization vector" $n$

$$
n_{\mu}=-\left[g_{\mu \nu}-\frac{p_{t_{\mu}} p_{t_{\nu}}}{m_{t}^{2}}\right] \frac{\sum_{\mathrm{spin}} \int d \Phi \bar{B} \Lambda_{+} \gamma_{5} \gamma^{\nu} B}{\sum_{\mathrm{spin}} \int d \Phi \bar{B} \Lambda_{+} B}
$$

where the spinor $B$ is defined such that the matrix element for $t\left(s_{t}\right) \rightarrow \ell+\cdots$ is expressed as $\bar{B} u_{t}\left(p_{t}, s_{t}\right), \Lambda_{+} \equiv \not p_{t}+m_{t}, d \Phi$ is the relevant final-state phase-space element, and $\sum_{\text {spin }}$ denotes the appropriate spin summation. $d \Gamma(t \rightarrow \ell X)$ for the amplitude (16) is given by

$$
\frac{1}{\Gamma_{t}} \frac{d \Gamma}{d x d \omega}=\frac{1+\beta}{\beta} \frac{3 B_{\ell}}{W} \omega\left[1+2 \operatorname{Re}\left(f_{2}^{R}\right) \sqrt{r}\left(\frac{1}{1-\omega}-\frac{3}{1+2 r}\right)\right]
$$


where $r \equiv\left(M_{W} / m_{t}\right)^{2}, W \equiv(1-r)^{2}(1+2 r), \omega \equiv\left(p_{t}-p_{\ell}\right)^{2} / m_{t}^{2}$, and $x$ is defined by the $t \bar{t}$ CM-frame lepton-energy $E_{\ell}$ and $\beta \equiv \sqrt{1-4 m_{t}^{2} / s}$ as

$$
x \equiv \frac{2 E_{\ell}}{m_{t}} \sqrt{(1-\beta) /(1+\beta)} .
$$

Note that eq.(174) holds very generally as long as the narrow-width approximation is applicable to both the top-quark and $W$-boson propagators.

\section{A2. Cross sections in $e \bar{e}-$ and $\gamma \gamma$-CM frames}

Calculations of cross sections are much simpler in $\gamma \gamma$-CM frame, while actual experimental measurements are performed in $e \bar{e}-\mathrm{CM}$ frame. Any observables expressed in these two frames are of course connected via a proper Lorentz transformation. We summarize here some necessary formulas.

Let us express the lepton energy and scattering angle in $e \bar{e}$-CM frame as $E$ and $\theta$, and those in the $\gamma \gamma$-CM frame as $E^{\star}$ and $\theta^{\star}$. Their relation is given by

$$
E^{\star}=\frac{E(1-\beta \cos \theta)}{\sqrt{1-\beta^{2}}}, \quad \cos \theta^{\star}=\frac{\cos \theta-\beta}{1-\beta \cos \theta},
$$

where

$$
\beta=(y-\tilde{y}) /(y+\tilde{y})
$$

$y$ and $\tilde{y}$ are the energy fractions of $e$ and $\bar{e}$ carried by the initial photon 1 and 2 , which appeared in eq.(54). The scattering angle is defined as the angle between the final-lepton $\ell$ and the initial-photon 1 .

Based on these formulas, we have

$$
\begin{aligned}
\frac{d \sigma}{d E d \cos \theta} & =\int d y d \tilde{y} \frac{d N(y)}{d y} \frac{d N(\tilde{y})}{d \tilde{y}} \frac{d \sigma}{d E d \cos \theta}(y, \tilde{y}) \\
& =\int d y d \tilde{y} \frac{d N(y)}{d y} \frac{d N(\tilde{y})}{d \tilde{y}} J(y, \tilde{y}) \frac{d \sigma}{d E^{\star} d \cos \theta^{\star}}(y, \tilde{y})
\end{aligned}
$$

where the Jacobian $J(y, \tilde{y})$ is given by

$$
J(y, \tilde{y})=\frac{\sqrt{1-\beta^{2}}}{1-\beta \cos \theta} .
$$

$d \sigma(y, \tilde{y}) /\left(d E^{\star} d \cos \theta^{\star}\right)$ is originally a function of $E^{\star}$ and $\cos \theta^{\star}$, but they have to be re-expressed in terms of $E$ and $\cos \theta$ in eq.(179) through the relations given in eq.(177). 
Finally let us show the integration region for $E$ and $\cos \theta$ in $d \sigma /(d E d \cos \theta)$. It is easy to confirm

$$
-1 \leq \cos \theta^{\star} \leq+1 \quad \Longleftrightarrow \quad-1 \leq \cos \theta \leq+1
$$

On the other hand, the upper and lower bounds of the energy are a bit more complicated. Since

$$
\frac{m_{t} r}{2} \sqrt{\frac{1-\beta^{\star}(y, \tilde{y})}{1+\beta^{\star}(y, \tilde{y})}} \leq E^{\star} \leq \frac{m_{t}}{2} \sqrt{\frac{1+\beta^{\star}(y, \tilde{y})}{1-\beta^{\star}(y, \tilde{y})}},
$$

where $\beta^{\star}(y, \tilde{y}) \equiv \sqrt{1-4 m_{t}^{2} /(y \tilde{y} s)}$, one obtains

$$
\frac{1}{2} m_{t} r \sqrt{\frac{1-\beta^{\star}(y, \tilde{y})}{1+\beta^{\star}(y, \tilde{y})}} \frac{\sqrt{1-\beta^{2}}}{1-\beta \cos \theta} \leq E \leq \frac{1}{2} m_{t} \sqrt{\frac{1+\beta^{\star}(y, \tilde{y})}{1-\beta^{\star}(y, \tilde{y})}} \frac{\sqrt{1-\beta^{2}}}{1-\beta \cos \theta} .
$$

Since the right(left)-hand side takes its maximum (minimum) for $y=\tilde{y}=y_{\max }$, we have

$$
\frac{1}{2} m_{t} r \sqrt{\frac{1-\beta_{\max }^{\star}}{1+\beta_{\max }^{\star}}} \leq E \leq \frac{1}{2} m_{t} \sqrt{\frac{1+\beta_{\max }^{\star}}{1-\beta_{\max }^{\star}}}
$$

for the energy integration in the $e \bar{e}$-CM frame, where $\beta_{\max }^{\star} \equiv \beta^{\star}\left(y_{\max }, \tilde{y}_{\max }\right)$.

\section{REFERENCES}

[1] CDF Collaboration : F. Abe et al., Phys. Rev. Lett. 73 (1994), 225 (hep-ex/9405005); Phys. Rev. D50 (1994), 2966; Phys. Rev. Lett. 74 (1995), 2626 (hep-ex/9503002);

D0 Collaboration : S. Abachi et al., Phys. Rev. Lett. 74 (1995), 2632 (hep-ex/9503003).

[2] D. Atwood, S. Bar-Shalom, G. Eilam and A. Soni, Phys. Rept. 347 (2001), 1 (hep-ph/0006032).

[3] ACFA Linear Collider Working Group Collaboration : K. Abe et al., "Particle physics experiments at $J L C$ ' KEK Report 2001-11 (hep-ph/0109166).

[4] I.F. Ginzburg, G.L. Kotkin, V.G. Serbo and V.I. Telnov, Nucl. Instrum. Meth. 205 (1983) 47. 
[5] D.L. Borden, D. Bauer and D.O. Caldwell, SLAC-PUB-5715, UCSB-HEP-9201.

[6] B. Grzadkowski and J.F. Gunion, Phys. Lett. B294 (1992), 361 (hep-ph/9206262).

[7] H. Anlauf, W. Bernreuther and A. Brandenburg, Phys. Rev. D52 (1995), 3803; ibid. D53 (1995), 1725 (Erratum) (hep-ph/9504424).

[8] G.J. Gounaris and G.P. Tsirigoti, Phys. Rev. D56 (1997), 3030, ibid. D58 (1998) 059901 (Erratum) (hep-ph/9703446).

[9] E. Asakawa, J. Kamoshita, A. Sugamoto and I. Watanabe, Eur. Phys. J. C14 (2000), 335 (hep-ph/9912373).

[10] E. Asakawa, S.Y. Choi, K. Hagiwara and J.S. Lee, Phys. Rev. D62 (2000), 115005 (hep-ph/0005313).

[11] R.M. Godbole, S.D. Rindani and R.K. Singh, Phys. Rev. D67 (2003), 095009 (hep-ph/0211136).

[12] E. Asakawa and K. Hagiwara, Report UT-ICEPP 03-04/KEK-TH-888 (hep-ph/0305323).

[13] S.Y. Choi and K. Hagiwara, Phys. Lett. B359 (1995), 369 (hep-ph/9506430)).

[14] M.S. Baek, S.Y. Choi and C.S. Kim, Phys. Rev. D56 (1997), 6835 (hep-ph/9704312).

[15] P. Poulose and S.D. Rindani, Phys. Rev. D57 (1998), 5444; ibid. D61 (1998), 119902 (Erratum) (hep-ph/9709225);

[16] W. Buchmüller and D. Wyler, Nucl. Phys. B268 (1986), 621.

[17] J.A.M. Vermaseren, "Symbolic Manipulation with FORM" version 2, Tutorial and Reference Manual, CAN, Amsterdam 1991, ISBN 90-74116-01-9.

[18] C. Arzt, M.B. Einhorn and J. Wudka, Nucl. Phys. B433 (1995), 41 (hep-ph/9405214). 
[19] T. Appelquist and J. Carazzone, Phys. Rev. D11 (1975), 2856;

J. Collins, F. Wilczek and A. Zee, Phys. Rev. D18 (1978), 242;

Y. Kazama and Y.P. Yao, Phys. Rev. D25 (1982), 1605.

[20] P. Poulose and S.D. Rindani, Phys. Lett. B452 (1999), 347 (hep-ph/9809203).

[21] D. Atwood and A. Soni, Phys. Rev. D45 (1992), 2405;

M. Davier, L. Duflot, F. Le Diberder and A. Rouge, Phys. Lett. B306 (1993), 411 ;

M. Diehl and O. Nachtmann, Z. Phys. C62 (1994), 397;

J.F. Gunion, B. Grzadkowski and X.G. He, Phys. Rev. Lett. 77 (1996), 5172 (hep-ph/9605326).

[22] B. Grzadkowski and Z. Hioki, Phys. Lett. B557 (2003), 55 (hep-ph/0208079); See also

B. Grzadkowski and Z. Hioki, Phys. Lett. B476 (2000), 87 (hep-ph/9911505); Phys. Lett. B529 (2002), 82 (hep-ph/0112361);

S.D. Rindani, Pramana 54 (2000) 791 (hep-ph/0002006).

[23] T. Takahashi and K. Ikematsu, Int. J. Mod. Phys. A15 (2000), 2599.

[24] Y.S. Tsai, Phys. Rev. D4 (1971), 2821; ibid. D13 (1976), 771(Erratum);

S. Kawasaki, T. Shirafuji and S.Y. Tsai, Prog. Theor. Phys. 49 (1973), 1656. 